\title{
VIDEOAULA: PRODUTO INFORMACIONAL PARA PROMOÇÃO DA AUTOAPRENDIZAGEM
}

Pedro Eduardo Galvão Cardoso - pegc.ca@gmail.com

UFPR, Departamento de Gestão da Informação

Avenida Prefeito Lothário Meissner, 632, Jardim Botânico

82.590-300 Curitiba, PR.

Maria do Carmo Duarte de Freitas - carmemk2@gmail.com

UFPR, Departamento de Gestão da Informação

Avenida Prefeito Lothário Meissner, 632, Jardim Botânico

82.590-300 Curitiba, PR.

Renan Dinis Pergher - renanpergher@hotmail.com

UFPR, Departamento de Engenharia Civil

Avenida Prefeito Lothário Meissner, 632, Jardim Botânico

82.590-300 Curitiba, PR.

Aline Fornari-fornarialine@gmail.com

UFPR, Departamento de Gestão da Informação

Avenida Prefeito Lothário Meissner, 632, Jardim Botânico

82.590-300 Curitiba, PR.

Resumo: O grande aumento de usuários na internet tem trazido vários recursos para as mídias digitais, como o compartilhamento de conhecimento por meio de cursos remotos e a oferta de cursos de graduações pelo ambiente virtual, pelo formato de vídeo. Com o cenário de isolamento social devido a pandemia (COVID-19), a adesão aos meios digitais para a educação foi unânime em todos os níveis educacionais, elevando a autoaprendizagem. Esta pesquisa tem como objetivo investigar a existência de diretrizes para a produção de vídeos como informação de modo a propiciar autoaprendizagem, considerando a demanda e êxito no ensino a distância da mesma maneira que presencialmente. Para o desenvolvimento deste estudo, parte-se da pesquisa bibliográfica sobre temas relacionados e busca por modelos existentes de elaboração de videoaulas. Em seguida, o passo a passo para quem disponibilizará o conhecimento (professor, técnico, conteudista) sem habilidade com a produção de vídeo, mas dispõe do conhecimento pedagógico e técnico do tema a ser partilhado. Apresenta-se a aplicação do vídeo como um produto de informação estratégico para a auto aprendizagem no projeto \#Soufpr, o qual propõe cursos no formato de MOOCs, de maneira prática a elaboração de videoaulas. A expectativa é que os professores realizem as videoaulas com caráter interativo, dinâmico e de fácil compreensão. A transmissão do conhecimento de forma online por vídeos, permitindo a expansão da área, colaborando muito com a comunidade $e$ imprescindível no momento de distanciamento social, porém, para que ocorra, há preocupação com todas as fases de elaboração do conteúdo a ser transmitido.

Palavras-chave: Videoaula. Autoaprendizagem. Produto informacional. Ensino a distância. Professor. 


\section{INTRODUÇÃO}

Com o cenário de isolamento social devido a pandemia do COVID-19, a adesão aos meios digitais para a educação foi unânime em todos os níveis educacionais e assim, consequentemente, elevou-se a promoção da autoaprendizagem. Isso também acabou fazendo com que muitas Instituições de Ensino Superior (IES), repensem a suas ofertas de cursos que até então eram ofertados presencialmente, agora são ofertados na modalidade a distância, por meio de vídeos. Porém, o fato desta ruptura do modelo tradicional presencial para a modalidade a distância, fez com que muitos professores se sentissem intimidados com o uso das tecnologias e principalmente na produção de vídeos.

De acordo com Jesus e Souto (2018), grande parte dos professores de que lecionam hoje ainda são considerados imigrantes digitais, pelo fato de que eles não nasceram nessa era tecnológica e nem mesmo receberam formação para a utilização da tecnologia para o uso pedagógico. Sendo assim, estes docentes têm dificuldades para produzir conteúdo pedagógico por meio das ferramentas tecnológicas.

Ainda conforme os autores, é necessário considerar que a escolha e/ou produção de um vídeo para fins educacionais não é uma tarefa simples. "Mesmo diante desses desafios, ao que parece, os professores têm se mobilizado na tentativa de produzir e/ou utilizar vídeos que possam contribuir com os processos de ensino e de aprendizagem, de forma particular na Educação a Distância” (JESUS; SOUTO, 2018, p. 49).

Nesta pesquisa, busca-se trazer o conceito das videoaulas associado a autoaprendizagem como um produto informacional, considerando que, dentro de um âmbito de grande acesso aos meios digitais, é imprescindível que a produção de videoaulas seja feita de modo com que todos que estejam assistindo façam um bom aproveitamento do conhecimento disposto.

Deste modo, é importante que professores e os conteudistas dispostos a partilhar conhecimento estejam aptos a realizarem suas aulas remotas da melhor maneira possível, buscando a efetividade na autoaprendizagem.

Considerando o vídeo como a maneira mais usada para isso, é importante haver diretrizes bem definidas para que esse meio digital possa passar o conteúdo de maneira íntegra e completa, servindo como um grande auxílio para aquele que irá aprender ao assistir.

\section{REVISÃO DA LITERATURA}

Com o crescimento do acesso a informações por meio da internet, a educação passa a ser desenvolvida dentro de meios digitais. Desta forma, uma tendência emergente são cursos em plataformas digitais que ofereçam conhecimento de fácil acesso aos usuários. Num cenário de menos de dez anos atrás, criam-se os Massive Open Online Courses - MOOCs, oferecendo conhecimento de forma aberta e gratuita.

O termo MOOC surge pela primeira vez após a iniciativa de George Siemens de disponibilizar seu curso Connectivism and Connective de forma não presencial abrigando assim um público de 2.300 pessoas. Apesar disso, a conceituação dos cursos massivos online têm diversas perspectivas.

McAuley, Stewart, Siemens e Cormier (2010) dizem que um MOOC é um curso on-line com a opção de inscrição gratuita e aberta, é uma oferta de currículo compartilhado pelo público. Já Subbian (2013), afirma que um MOOC é um curso gratuito baseado na web com registro aberto, currículo compartilhado publicamente e resultados de aprendizado abertos.

Além do conceito aberto e de fomentar a integração do conhecimento a utilização dos MOOCs. Siemens (2013), afirma que os MOOCs "oferecem um meio termo para o ensino e 
aprendizagem entre o ambiente de sala de aula altamente organizado e estruturado e a caótica rede aberta de informações fragmentadas. Numa sala de aula tradicional ou curso on-line, designers e educadores de aprendizagem estruturam as leituras recursos de aprendizagem, palestras e atividades dos alunos. Como resultado, o aprendizado é direcionado a objetivos e resultados claramente articulados. O educador fornece molde e direciona a experiência de aprendizagem, formando grupos e fornecendo avaliações, focos de tarefas ou diretrizes"

\subsection{Autoaprendizagem - Conceito e aplicação}

Dentro de um cenário de maior acesso a informações por meios digitais, vemos como a aprendizagem tem um potencial de expansão por tais meios e, dessa forma, um processo de aquisição de conhecimento por meio de um MOOC entra dentro do conceito de autoaprendizagem. O qual junta a aprendizagem convencional que antes era dependente de um professor, o qual tem o papel de tutor de seus alunos, mas agora, de forma massiva, não pode atender a todos, os quais têm para si a atribuição de aprender por conta própria absorvendo o conhecimento disponibilizado.

Esta forma de aprendizagem já tem sido citada por alguns autores, como Nyhan (1996, p. 48) o qual afirma que:

Pode considerar-se como uma aprendizagem aprofundada e alargada, mais do que uma aprendizagem de procedimentos e regras, pois este conhecimento aprofundado, assim como o domínio de tarefas e situações específicas, originam a apropriação de princípios e processos genéricos que podem ser transferidos para enfrentar qualquer outra área específica.

Já Knowles, (1975, 1980, p. 88), por sua vez, define autoaprendizagem como:

Um processo no âmbito do qual os indivíduos tomam a iniciativa, com ou sem a ajuda de outros, diagnosticando as suas necessidades de aprendizagem, formulando os seus objetivos neste âmbito, identificando os recursos humanos e materiais que necessitam para aprender, escolhendo e implementando estratégias de aprendizagem apropriadas e avaliando os resultados dessa aprendizagem.

Os autores Van Berkel \& Schmidt (2000, p.232) trazem, também, uma visão diferente mas importante sobre a autoaprendizagem, atrelando-a ao trabalho em grupo, onde realizam um trabalho de pesquisa real, motivando os alunos a estudarem a partir de alguma problemática, "[...] os alunos trabalham pequenos grupos sobre esses problemas, e, enquanto discutem sobre isso, formulam objetivos para a autoaprendizagem".

Os estudos dos autores trazem alguns fatores de relevância no processo de autoaprendizagem, de onde podem-se fazer algumas considerações:

- A iniciativa é do aluno em buscar o conhecimento;

- A absorção de conhecimento através da autoaprendizagem também possibilita a futura transferência do que foi aprendido;

- Os alunos tendem a se reinventar para terem melhores resultados com a autoaprendizagem, visto que a o professor ou tutor do curso não tem toda a atenção que teria em uma sala de aula comum, os mesmos recorrem, por exemplo, a trabalhar em grupo.

\subsection{Produto Informacional}

Para se definir Produto Informacional pode-se dividir os termos Produto e Informação. Um produto é definido por (SEMMENIK, 1995) como "um conjunto de atributos tangíveis e 
intangíveis, que proporciona benefícios reais ou percebidos, com a finalidade de satisfazer as necessidades e os desejos do consumidor". (p.260). Já a informação é descrita por Le Coadic (1996): "Como conhecimento inscrito (gravado) sob a forma escrita (impressa ou numérica), oral ou audiovisual. É um significado transmitido a um ser consciente por meio de uma mensagem inscrita em suporte espacial-temporal: impresso, sinal elétrico, onda sonora, etc."

A junção desses termos resulta no termo PRODUTO DE INFORMAÇÃO, e esse termo é definido por Silva, Santos \& Freitas $(2008$, p.2) como "o resultado tangível de todo processo de gestão da informação (coleta, análise, tratamento, disseminação e armazenamento) que propicia um benefício por meio de sua utilização, visando sempre atender as necessidades identificadas."

Outro termo importante para a compreensão de produto informacional é serviços de informação. O mesmo é caracterizado por Rozados (2004) e adaptado por Borges (2007 p.117): "Todo processo de auxílio ao usuário na busca de informação ou na satisfação de suas necessidades informacionais". A partir daí, pode-se compreender um produto de informação como "estruturas de informacionais resultantes de serviços" (ROZADOS, 2004, p.118). Uma propriedade relevante e que diferencia os produtos de serviços é a tangibilidade, presente nos produtos informacionais por meio de suas características de apresentação, forma e modelo.

Pode-se analisar que um pequeno ciclo entre Uso - Armazenamento - Recuperação - Reuso. Isso indicia que um produto informacional não segue o padrão consumista de produto que tem consumo e descarte, mas, ao contrário disso, tem inúmeros usos ao longo do tempo. Essa afirmação é defendida por Galvão (1999), e está presente em Silva, Santos \& Freitas (2008, p.3): "Um software ou um produto audiovisual é utilizado inúmeras vezes sem que se deprecie ou perca valor".

\subsection{A utilização de vídeos na educação}

Com base no contexto de expansão da educação dentro dos meios digitais e, visando o aumento da eficiência de ensino aos alunos, vê-se a necessidade da integralização de recursos tecnológicos no ambiente de aula, principalmente a utilização de vídeos. A eficiência do uso de um recurso audiovisual perante aos alunos pode ser observado como descrito por Bleicher (2009) considerando que $40 \%$ das pessoas respondem melhor a estímulos audiovisuais do que a texto, onde o cérebro humano processa esses estímulos 60 mil vezes mais rápido do que a velocidade com que ele processa palavras.

No Brasil, a inserção do vídeo como um instrumento didático se dá na segunda metade da década de 80 e a sua popularização como instrumento de dinamização desse processo só se verificará na década de 90 (LIMA, 2001). Além do caráter comunicativo que é associado ao vídeo, Férres (2001) afirma que o vídeo é um meio comunicacional e de ensino.

Porém, utilizar-se deste recurso necessita de uma grande preparação, pois o vídeo torna-se muito mais do que uma simples tecnologia, caracterizado novamente por Férres (1998). Para a escola ele é um desafio. Desta forma, fica nítida a importância de ter orientações para o corpo docente ao se utilizarem de vídeos como meio de transferência de conhecimento aos alunos.

As videoaulas tem ganhado destaque e defesa de autores. Schneider (2014, p. 90) argumenta: "Muitas publicações atuais falam defendem o uso de vídeos na educação e apresentam propostas de utilização e avaliação [...]”. Ou seja, essa forma de ensino já vinha se popularizando por anos atrás e aumentou de maneira brusca no início do ano de 2020 , em consequência da pandemia de COVID-19. Mesmo antes do cenário atual, já era visto o grande potencial de compartilhamento de conhecimento, bem como mencionado pelos autores Spanhol e Spanhol (2009, p. 3): “"a videoaula é um recurso que impulsiona a disseminação do conhecimento". 


\subsection{Metodologias para a produção VideoAula}

Há várias metodologias relacionadas a produção de videoaulas, porém, com várias pesquisas na área, não se pode eleger um modelo perfeito, mas, há como eleger elementos presentes em pesquisas e que compõem diretrizes para a produção de videoaulas.

Os autores Spanhol e Spanhol (2009), trazem diretrizes mais genéricas que, de certa forma abrigam a de outros autores, porém, quando se tratando de produção (pós e pré), é algo muito abrangente.

Desta forma, pode-se dividir a pré-produção em partes como o planejamento e principalmente o roteiro, diretriz abordada por vários dos autores do quadro. Ambas são muito importantes para que a videoaula seja feita com um caminho prévio a ser seguido, garantindo assim que a aula será gravada sem desviar o assunto a ser tratado. Há passos que são abrigados por todos os autores, como a gravação a edição/sincronização (abrigados, também, na etapa de produção). Esses passos são essenciais para a construção da videoaula e recebem uma atenção a mais, visto que para o espectador, o resultado que será visto, é o vídeo propriamente dito.

Para uma melhor evolução nas etapas, o professor que ministra o curso, necessita da utilização de metodologias. Isso é defendido por autores como Mussio (2014), Spanhol e Spanhol (2009) e Taú et al., (2014), onde "relatam que para a correta e efetiva utilização de vídeos no processo educativo, os mesmos necessitam de metodologias para a sua produção" (MARCELINO, 2018, p.15), ou seja, é preciso que se tenha a metodologia correta para o cumprimento de determinada atividade para que possa haver a continuidade das outras etapas e, assim, garantir que o resultado final seja bem sucedido. Anteriormente a busca do desenvolvimento de técnicas é preciso analisar os caminhos a serem tomados para a produção, pois só assim pode-se definir as habilidades a serem desenvolvidas em determinada etapa, ressaltando a importância a ser tomada.

Para o início da elaboração pode-se fazer alguns questionamentos, os quais são abordados também por Schneider (2014): Qual o assunto que será abordado no vídeo? Qual será o texto? Como esse material será capturado, com uma filmadora, câmera fotográfica ou celular? Serão capturadas imagens em movimento ou uma sequência de fotografias? Onde o filme acontece? É necessário trilha sonora? Há narrador? A partir do momento em que esses questionamentos iniciais forem respondidos, segue-se para o desenvolvimento das técnicas que serão usadas e, enfim, para a prática das etapas previamente definidas.

\section{MATERIAIS E MÉTODOS}

De acordo com Köche (2015), a investigação científica se inicia com a identificação de uma dúvida, de uma pergunta que ainda não possui resposta e com o reconhecimento de que o conhecimento existente é insuficiente para esclarecer essa dúvida. A necessidade de construir uma resposta para essa dúvida que ofereça provas de segurança e confiabilidade que justifiquem a crença de ser uma boa resposta.

Quanto aos objetivos, o relatório é uma pesquisa bibliográfica. Segundo Gil (2008, p. 50), "a pesquisa bibliográfica é desenvolvida a partir de material já elaborado, constituído principalmente de livros e artigos científicos". Ainda de acordo com o autor, ainda que em quase todos os estudos seja exigido algum tipo de trabalho desta natureza, existem pesquisas desenvolvidas exclusivamente a partir de fontes bibliográficas.

Sendo assim, o processo metodológico ocorreu por meio de pesquisas nas seguintes bases de dados e periódicos: Scielo, Google Acadêmico e no Portal de Periódicos da CAPES. Para o 
processo de busca foi utilizado as palavras MOOC AND educação, vídeo AND educação e vídeo AND informação. Partido de textos encontrados com estas pesquisas, busca-se procurar entre os autores mais citados maiores informações a serem acrescidas no texto.

Considerando a pesquisa laboratorial, a mesma foi realizada no Grupo de Pesquisa em Ciência, Informação e Tecnologia, da Universidade Federal do Paraná, onde houve a elaboração de um curso MOOC, de forma experimental dentro do projeto \#Soufpr. Nesse sentido, Gil (2008) afirma que, o experimento é o melhor exemplo de pesquisa científica. Pois, consiste em determinar um objeto de estudo, seleciona as variáveis que seriam capazes de influenciar, define as formas de controle e de observação dos efeitos que a variável produz no objeto.

Com base nestas informações, foi dado a oportunidade da aplicação prática durante a pesquisa sendo realizada, possibilitando também oportunidade de pesquisa assim que se observava problemas práticos que ocorriam no andamento do projeto.

\section{RESULTADOS E DISCUSSÃO}

Os procedimentos que ocorreram para se chegar nos primeiros resultados acontecem a partir da criação de um projeto desenvolvido dentro do Grupo de Pesquisa em Ciência, Informação e Tecnologia, reunindo alunos de graduação, mestrado e doutorado de diversos cursos da UFPR.

Tal projeto é denominado como \#Soufpr e, o mesmo abre uma afluente que objetiva a elaboração de uma plataforma para a publicação de cursos em formato de MOOC, buscando compartilhar o conhecimento de pesquisas desenvolvidas na universidade para outras pessoas, sendo elas do ambiente acadêmico ou não e de forma gratuita. O financiamento deste se dá por meio da CAPES e da Fundação Araucária as quais disponibilizam bolsas de iniciação científica aos alunos participantes, além da própria universidade que paga o salário dos professores envolvidos.

Para a construção da plataforma houveram várias etapas, e a elaboração propriamente dita não foi o primeiro passo tomado. Inicialmente começou a ser feito o primeiro curso que seria disponibilizado futuramente e o tema abordado no mesmo é "Gestão Lean na Construção Civil". O tema foi escolhido a partir de pesquisas previamente elaboradas dentro da área de gestão e de construção civil onde as mesmas seguem uma linha de pesquisa que vem sendo incrementada por anos trazendo maior credibilidade para o tema do curso.

No curso modelo, a professora que ministrou era mestranda em Engenharia Civil e coloca sua dissertação de mestrado apoiada em toda a pesquisa desenvolvida voltada ao curso, contando com todo o amparado de sua orientadora.

Numa proposta de ocorrerem cursos futuros, a exigência seria de que todos os próximos professores de cursos de fato fossem do corpo docente da universidade e, também, sejam pesquisadores de algum assunto e, com essa linha de pesquisa concretizada possa partir para uma elaboração de um curso, reforçando a credibilidade mencionada anteriormente..

Com a base teórica já estruturada, parte-se para parte mais prática, chegando então a produção de vídeos. Esta produção passa por um processo que abriga desde a elaboração de materiais até a criação material da plataforma para a publicação do mesmo. Foi elaborada por uma pessoa encarregada de estar operando a câmera, outra para ajustes de equipamentos e aquele que ministra o curso.

Quando se tratando da produção audiovisual, há uma subdivisão em várias etapas para chegar no resultado de um vídeo completo e essa subdivisão acontece devido a variedade de elementos que necessitam de atenção na elaboração de um material que, apesar de ter toda sua 
base teórica bem fundamentada necessita ter uma abordagem tão confiável quanto a teoria, deixando o espectador (que se torna um aluno) confiante e interessado a usufruir do conhecimento ali disposto.

Para esta produção, usou-se como referencial outros cursos já disponíveis, notando elementos gerais como a gravação e a exibição de conteúdo em imagens (slides) e, a partir disso, pode-se dar início a parte de filmagem das aulas do curso, que ocorreram em estúdio, contando com elementos como: microfone de lapela, dando mais qualidade ao som; fundo de pano verde, visando uma posterior edição do vídeo; câmera de boa qualidade, deixando a imagem nítida. Contando com esses elementos, pode-se gravar todas as aulas do curso, passando assim para o próximo passo, que foi a edição e tratamento do vídeo.

A etapa de edição é de grande relevância pois é ali em que informações serão montadas para posterior apresentação e, neste curso inicial, contou com apenas uma pessoa em prática de edição. Para que haja a edição do vídeo é necessário um software a ser utilizado pelo editor e, neste caso, por não contarmos com nenhuma licença de programas pagos, optou-se pela utilização de um software gratuito intitulado "DaVinci Resolve".

Com a escolha e instalação do software, deram-se os primeiros passos de edição, a qual ocorreria com referência em modelos de outros MOOC's, analisando elementos como o tempo destinado a cada aula do curso, tempo de vídeo que era entre 3 a 5 minutos em vários cursos, a forma de colocar textos ao fundo e até mesmo o posicionamento da pessoa que está falando.

Outro elemento importante dentro do vídeo, é a introdução, onde, após discussões para decidir o conteúdo da mesma, decidiu-se que contaria com o nome do projeto e, também, com o título do curso acompanhando com o assunto da aula editada. No fim de cada vídeo também elaborou-se uma passagem de créditos, abrigando o nome de todos os colaboradores em suas respectivas funções e mostrando também a logo de Creative Commons que faz referência ao fato de ser uma obra de conhecimento compartilhado e de possível expansão.

Após a edição, retoma-se a atenção a plataforma, onde é feita, de fato, a elaboração da mesma. Neste caso, utilizou-se a plataforma Moodle base para montagem e a hospedagem é feita em um servidor que se encontra dentro do ambiente físico do grupo de pesquisa, sem suporte para acessos em grande massa, visto que o curso inicial tinha um caráter de teste e não contaria com um número exorbitante de acessos.

Com a plataforma já feita e em funcionamento, foi possível adicionar todos os vídeos previamente editados na mesma, mas, os vídeos dentro da plataforma contariam também com atividades interativas por meio do recurso "H5P". Desta forma, a pessoa que fez o conteúdo do curso fica atribuída com a realização dessas atividades, proporcionando para o usuário e aluno uma experiência ampla de aquisição de conhecimento.

O curso tomava sua forma final e estava pronto para ser usado e, para tanto, foi usado como parte complementar de uma matéria do curso de Engenharia Civil trazendo os alunos como usuários para a plataforma, os quais puderam trazer as primeiras impressões do ambiente virtual. Neste feedback pode-se identificar resultados positivos, dando o contraste de como toda a preocupação na realização de cada etapa do curso e da plataforma foram reconhecidos pelos usuários e considerando possíveis pontos a melhorar.

\section{CONSIDERAÇÕES FINAIS}

Esta pesquisa tem potencial de contribuição dentro da produção de videoaulas, visto que traz procedimentos metodológicos sintetizados para as mesmas e, também, por abrigar uma experiência prática. Há a possibilidade de ser usada como base para elaboração de uma metodologia de videoaula, principalmente em um curso MOOC, mas, também, pela 
abrangência geral do assunto de videoaulas, pode-se expandir a aulas gravadas em outro modo de curso. A pesquisa também tem pode servir de base ou inspiração para aprofundamento em áreas relacionadas às videoaulas, visto que essa área está aumentando muito e as pesquisas sobre o tema (ou relacionadas) tendem a aumentar, fortalecendo o ensino remoto e o tornando cada vez mais acessível.

Para o aprendizado acadêmico, esta pesquisa teve grande relevância, considerando que houve, primordialmente, uma captura teórica de grande relevância, captando vários conceitos e aprofundando o conhecimento sobre pesquisas de videoaulas e suas diretrizes e, posteriormente, tendo a parte prática também, a qual permitiu realizar a aplicação de conceitos teóricos e buscar resultados dos mesmos.

Com a descoberta de novos conceitos e com a leitura de trabalhos de vários autores que estão referenciados na pesquisa, a amplitude de conhecimento adquirido têm grande impacto nas matérias da graduação, possibilitando a integração do conhecimento adquirido na sala de aula com o que foi adquirido através da pesquisa, dando um grande apoio na formação acadêmica.

\section{Agradecimentos}

Agradecimento a equipe do GPCIT, laboratório de gestão de informação da UFPR, ao PPGECC e seus professores e demais estrutura da Universidade Federal do Paraná. Também a CAPES, CNPQ e a Fundação Araucária - Governo do Estado do Paraná, pelo apoio e financiamento da pesquisa.

\section{REFERÊNCIAS}

BLEICHER, Sabrina. A influência dos novos media no design editorial: estudo do projecto gráfico da Folha de S. Paulo. 2009. 135 f. Dissertação (Mestrado em Estudos Editoriais) Departamento de Línguas e Culturas, Universidade de Aveiro, Aveiro, 2009. Disponível em: https://ria.ua.pt/bitstream/10773/2820/1/2009001211.pdf. Acesso em: 18 fev. 2020.

BORGES, Mônica Erichsen Nassif. O essencial para a gestão de serviços e produtos de informação. Revista Digital de Biblioteconomia e Ciência da Informação, Campinas, v. 5,

n. $1, \quad$ p. 115-128, jul. 2007. Disponível em:

https://brapci.inf.br/_repositorio/2010/05/pdf_c394c36e4d_0010623.pdf. Acesso em: 06 ago. 2020.

FERRÉS, J. Pedagogia dos meios audiovisuais e Pedagogia com os meios audiovisuais. In: SANCHO J. Maria (Org.) Para uma Tecnologia Educacional. Trad. Beatriz Affonso Neves. Porto Alegre: Artes Médicas, 2001.

GALVÃO, Alexander $\mathrm{P}$. Da convergência tecnológica à convergência empresarial: o audiovisual como espaço de acumulação na era da digitalização. 1999. Universidade Federal do Rio de Janeiro, Rio de Janeiro.

GIL, A. C. Métodos e Técnicas de Pesquisa Social. 6. ed. São Paulo: Atlas, 2008.

JESUS, R. P. S.; SOUTO, D. P. Cálculo III com o uso de Vídeos na Educação a Distância. COINSPIRAÇÃ̃_Revista de Professores que Ensinam Matemática. v. 1 n. 2, jul-dez. 2018. Disponível

em: 
http://sbemmatogrosso.com.br/publicacoes/index.php/coinspiracao/article/view/30 . Acesso em: 31 jul. 2020.

KÖCHE, J. C. Fundamentos de metodologia científica: teoria da ciência e iniciação à pesquisa.34. Ed. - Petrópolis, RJ. Vozes, 2015.

LE COADIC,Y.F. A ciência da informação. Brasília, DF, Briquet de Lemos. 1996.

LIMA, Artemilson Alves de. O uso do vídeo como um instrumento didático e educativo: um estudo de caso do CEFET-RN. 2001. Florianópolis, 140 f. Dissertação (Mestrado em Engenharia de produção) - Programa de Pós-Graduação em Engenharia de Produção. UFSC, 2001. Disponivel em: https://repositorio.ufsc.br/xmlui/handle/123456789/79843. Acesso em: 14 abr. 2020.

MARCELINO, Jean Douglas. Vídeo-aulas para apoiar o processo ensino-aprendizagem sobre funções da disciplina de algoritmos e lógica de programação. 2018. 64 f. Dissertação (Mestrado em Ciências, Matemática e Tecnologias) - Universidade do Estado de Santa Catarina, Joinville, 2018.

MCAULEY, Alexander; STEWART, Bonnie; SIEMENS, George; CORMIER, Dave. The MOOC Model for Digital Practice. University of Prince Edward, 2010. Disponível em: https://www.oerknowledgecloud.org/archive/MOOC_Final.pdf. Acesso em: 14 fev. 2020.

NYHAN, B. Desenvolver a capacidade de aprendizagem das pessoas: Perspectivas europeias sobre a competência de auto-aprendizagem e mudança tecnológica. Caldas da Rainha: Eurotecnet, 1996.

PERGHER, R. D.; FREITAS, M. C. D.; SILVA, H. O.; ISHIDA, C. Y. Rede de colaboração em educação aberta para engenheiro e arquiteto - disseminação de pesquisa sobre sustentabilidade. In: XLVII COBENGE, 2019, Fortaleza. Artigo. Fortaleza, 2013. Disponível em:

http://www.abenge.org.br/sis_submetidos.php?acao=abrir\&evento=COBENGE19\&codigo $=\mathrm{C}$ OBENGE19_00090_00002616.pdf. Acesso em: 06 ago. 2020.

ROZADOS, H. B. F. Indicadores Como Ferramenta Para Gestão de Serviços de Informação Tecnológica. 2004. 239 f. Tese (Doutorado em Ciência da Informação) Universidade Federal do Rio Grande do Sul, Porto Alegre, 2004. Disponível em: https://www.lume.ufrgs.br/handle/10183/5668. Acesso em: 18 jul. 2020.

SCHNEIDER, C. K. Parâmetros visuais como apoio à produção de vídeos educacionais para o ensino de ciência e tecnologia no contexto da mobilidade e conectividade. 2014. 164 f. Dissertação (Mestrado Profissional Em Educação E Tecnologia) - Instituto Federal Sul-RioGrandense, Pelotas, $2014 . \quad$ Disponível em: http://biblioteca.ifsul.edu.br/pergamum/anexos_sql_hom81/00000c/00000c5f.pdf. Acesso em: 18 maio 2020.

SIEMENS, George. Massive Open Online Courses: Innovation in Education? In: MCGREAL, Rory; KINUTHIA, Wanjira; MARSHALL, Stewart. Open Educational Resources: Innovation, Research and Practice. Vancouver: Commonwealth Of Learning And Athabasca University, 2013. p. 268. Disponível em: https://www.oerknowledgecloud.org/archive/pub_PS_OERIRP_web.pdf\#page=31. Acesso em: 12 fev. 2020. 
SILVA, R. A. et al. Reflexão teórica e conceitual sobre produto informacional e produto de informação. In: VIII SEPROSUL - SEMANA DE ENGENHARIA DE PRODUÇÃO SULAMERICANA, 2008, Bento Gonçalves, p. 1-7. Disponível em: https://pt.scribd.com/doc/37203829/Reflexao-teorica-e-conceitual-sobre-produto-informacionalou-produto-de-informacao. Acesso em: 08 maio 2020.

SIVAMUNI, K.; BHATTACHARYA, S. Assembling pieces of the MOOCs jigsaw puzzle. In: Innovation and Technology in Education (MITE), 2013 IEEE International Conference in MOOC. IEEE, 2013. p. 393-398. Disponível em: https://www.researchgate.net/publication/271550962_Assembling_pieces_of_the_MOOCs_jigsa w_puzzle. Acesso em: 17/02/2020.

SPANHOL, G. K.; SPANHOL, F. J. Processo de Produção de Vídeo-Aula. Santa Catarina: [s.n.], 2009. Disponível em: https://www.seer.ufrgs.br/renote/article/download/13903/7812 . Acesso em: 02/08/2020.

SUBBIAN, Vignesh. Role of MOOCs in integrated STEM education: A learning perspective. In: INTEGRATED STEM EDUCATION CONFERENCE (ISEC),Princeton, 2013. p. 1-4. Disponível em: https://www.researchgate.net/publication/261260110_Role_of_MOOCs_in_integrated_STEM_e ducation_A_learning_perspective. Acesso em: 12 fev. 2020.

\section{VIDEOAULA: INFORMATIONAL PRODUCT FOR THE PROMOTION OF SELF-LEARNING}

Abstract: The huge increase in users on the internet has brought several resources to digital media, such as knowledge sharing through remote courses and the offer of undergraduate courses through the virtual environment, through the video format. With the scenario of social isolation due to the pandemic (COVID-19), adherence to digital media for education was unanimous at all educational levels, increasing self-learning. This research aims to investigate the existence of guidelines for the production of videos as information in order to provide self-learning, considering the demand and success in distance learning in the same way as in person. For the development of this study, we start from the bibliographic research on related topics and search for existing models for the elaboration of video classes. Then, step by step for those who will make the knowledge available (teacher, technician, content writer) without skill with video production, but have the pedagogical and technical knowledge of the topic to be shared. The application of video is presented as a strategic information product for self-learning in the \#Soufpr project, which proposes courses in the format of MOOCs, in a practical way the preparation of video classes. The expectation is that the teachers carry out the video lessons with an interactive, dynamic and easy to understand character. The transmission of knowledge online through videos, allowing the expansion of the area, collaborating a lot with the community and essential in the moment of social distance, however, for it to occur, there is concern with all stages of preparation of the content to be transmitted.

Keywords: Video class. Self-learning. Informational product. Distance learning. Teacher. 